\title{
High-Precision Determination of the Purity of Organic Materials by Differential Scanning Calorimetry
}

\author{
Kiyo Yamamoto*, Michihiko Momota**, Hisayo Kitamura* and Kusuo NaRIta* \\ *School of Pharmaceutical Sciences, University of Shizuoka, Yada, Shizuoka 422, Japan \\ **Rigaku Corporation, Akishima, Tokyo 196, Japan
}

\begin{abstract}
The technique described here improves the purity determination of organic compounds through the use of the differential scanning calorimetry (DSC). The dispersion in the slope of a DSC curve has been observed to have great impact on the measurement results. The causes of this dispersion are the bulkiness and high thermal resistance of organic compounds. The sampling method is therefore directly related to the characteristics of the generated DSC curve. Pressing the sample onto the bottom of a sealed sample container improves the reproducibility of the slope. Sample was pressurized at $100 \mathrm{kgf}$ for one minute by using a correction jig. The slope of the DSC curve representing a pure sample was obtained from the thermal conductivity of the sample. This value was used for calibration during computation of the sample purity. When conducted in this manner, the determination of purity based on the DSC method yields values with a maximum error of $0.1 \mathrm{~mol} \%$ for samples with a minimum purity of $99 \mathrm{~mol} \%$.
\end{abstract}

Keywords Purity determination, precise determination, differential scanning calorimetry, thermal resistance, container mold

An important concern of analytical chemistry is the determination of the purity of organic compounds. Most techniques that are currently being employed in instrumental analysis involve the analysis of a given sample in comparison with a standard sample. Advances in analytical instrumentation require an extremely high degree of purity warranty for standard samples. The determination of purity is thus important not only for laboratory use, but also for a broad spectrum of applications in which the quality warranty of reagents and drugs is vitally important. There is increasing need for standard samples with purity warranty and for more accurate determination methods that are easy to use.

The most desirable method to determine the purity is by a direct quantitative determination of the main constituent in a given sample. However, in many situations in chemical analysis, especially those involving organic compounds, it is difficult to achieve an error rate of $0.5 \%$ or less. ${ }^{1}$ The purity of a sample is conventionally determined by subtracting the sum of its trace impurity constituents from $100 \%$. Although this method provides an accurate purity value when the identity of the impurities involved is known, it is not accurate in situations when unknown impurities exist. The method is thus considered to be inaccurate. The thermal determination method, on the other hand, offers significant advantages, since it is capable of a direct quantitative determination of the main constituent in a sample. However, a simple determination of the sample purity through differential scanning calorimetry (DSC) is subject to a wide margin of error due to thermodynamic constraints ${ }^{2,3}$ as well as the variability inherent in measurement operations. Therefore, the DSC method is rarely used by itself to warrant purity.

The authors have already investigated purity determination techniques that are based on the melting point lowering method. ${ }^{4}$ They used a photometric melting point determination to observe how a sample melts, and then computed the sample purity. The purpose of this paper is to describe a technique for obtaining purity values that are reliable when tested by the DSC method.

The concentration of impurities in a sample can be determined by the van't Hoff's equation. DSC is used to record the fraction $(F)$ of a sample that has melted, as well as the change in the temperature $(T)$ that occurred during the melting process. The quantities $1 / F$ and $T$ are linearly related. It is known that when $1 / F=0, T$ indicates the melting point of a pure substance, and that when $1 / F=1, T$ indicates the melting point of a sample. The difference between these temperatures represents a decline in the melting point of the sample. Because DSC determinations are conducted dynamically, the thermal resistances of both the container and the sample give rise to a temperature difference due to heat conduction. Consequently, even a pure substance, when subjected to a DSC determination, produces a fusion curve that has an apparent slope. Hence, the temperature based on a sample DSC curve was calibrated by the DSC curve of a pure substance that constitutes the sample; the correct slope of the DSC curve is thus 
obtained for the substance, which is essential for an accurate determination of the purity of that substance.

In order to measure the DSC curves of samples with a high degree of reproducibility, the following procedure was used. After a sample was pressed onto the bottom of the sample container, an accurate correction value was obtained by computing the slope of the sample's DSC curve in terms of its thermal conductivity. The obtained purity of this manner is accurate with a high degree of reproducibility. This method is capable of determining the purity of a $99 \mathrm{~mol} \%$ sample with a maximum error of $0.1 \mathrm{~mol} \%$.

\section{Experimental}

\section{Equipment and apparatus}

TAS100 and 8230 furnace (Rigaku Co.) were used for DSC. An NEC PC-9801VX computer was used for data processing, and Rigaku's Puritas 3 program was used to compute the results.

A sealed container designed to hold liquid was used as the sample container. The sample was covered with a thin aluminum plate $4 \mathrm{~mm}$ in diameter. The apparatus for molding samples comprised of an upper piece, a lower piece, and a collar. Figure 1 shows details of this apparatus. The upper piece was provided with a $2 \mathrm{~mm}$ long rod, having a $4.4 \mathrm{~mm}$ outer diameter. The collar was equipped with a hole measuring $4.7 \mathrm{~mm}$ in inner diameter. The rod attached to the lower piece fitted snugly into the interior of the hole. Handpress A-1 (Matsushita Dendokogu Co.) was used as a molding apparatus to press the sample. The pressure applied to the sample to mold it was measured with a Tanita 109 system.

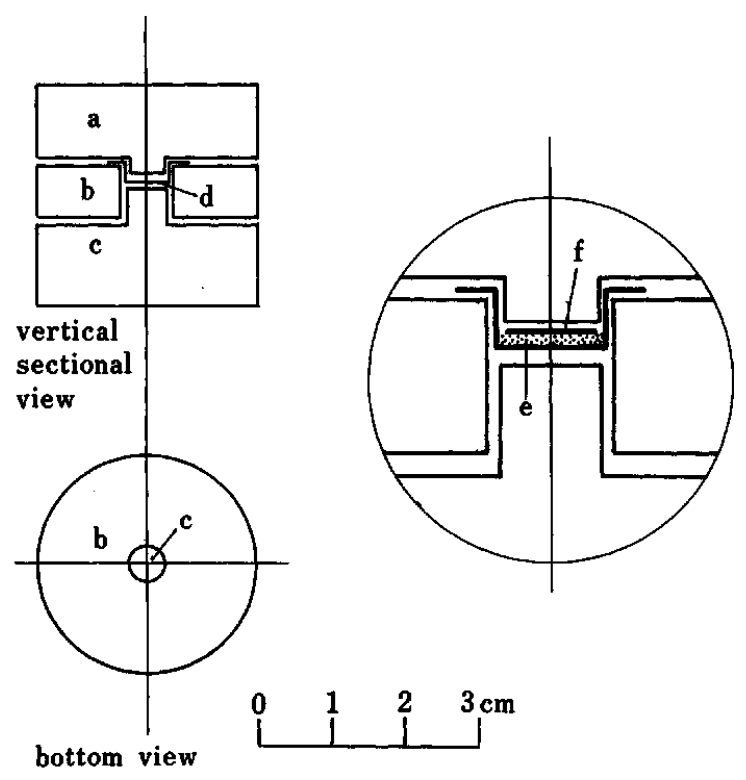

Fig. 1 Mold of container: a, upper piece; b, collar; c, bottom piece; $d$, sample container; $e$, sample; $f$, thin aluminum plate.

\section{Reagents}

Indium and tin standard samples ( $99.999 \%$ pure) were made by Rare Metal Co., Ltd. A phenacetin thermal analysis purity set (NBS SRM 1514) and organic microanalysis standard samples made by Kishida Chemical Co., Ltd., were used.

Pure phenacetin was obtained by purifying a Japanese Pharmacopoeia-grade product by recrystallization processes in water, methanol and ethanol. The substance was recrystallized twice in each solvent in the given order. After undergoing the recrystallization; the substance was dried at $80^{\circ} \mathrm{C}$ for $12 \mathrm{~h}$ under normal pressure. The melting point and the DSC curve were determined at each stage of recrystallization; the points where they did not change were made as the end point of purification.

Mixed samples were prepared as follows:

Minor constituents were dissolved with one drop of methanol. As the mixture was ground, the major constituent was gradually added. The resulting specimen was dried at ordinary temperature in a desiccator containing silica-gel.

\section{Procedures}

The tare of the aluminum plate and of the sample container was weighed; then, 2 to $3 \mathrm{mg}$ of a sample were placed in the container, and the sample was covered with an aluminum plate. The sample was then placed in a molding apparatus and pressed with a $100 \mathrm{kgf}$ load for $1 \mathrm{~min}$. Any portion of the sample that protruded from the plate was wiped off. Next, the sample container was placed on a balance so as to measure the sample amount. Finally, the sample was sealed under a stream of nitrogen gas, and left undisturbed for several hours.

For DSC the temperature was measured from $30 \mathrm{~K}$ below the melting point of the sample to $10 \mathrm{~K}$ above it at a rate of $1 \mathrm{~K} / \mathrm{min}$. The sample purity was calculated using the purity computation program.

\section{Results and Discussion}

\section{Causes of error}

To obtain reliable purity measurement values, the authors investigated the possible causes of dispersion in the purity values. It was found that neither the actual or molecular weight of a sample, nor any equipment constants, contributed significantly to the magnitude of the error.

The most probable source of error was found to be the variation in the slope of the DSC curve. Table 1 shows the changes in purity values (generated by the purity computation program) effected by a shift in the calibration slope values (ranging from -0.0418 to -0.1674 $\mathrm{mJ} / \mathrm{s}^{2}$ ) with the DSC curve obtained by using phenacetin in the thermal analysis purity set.

The purity of phenacetin was determined by using a single DSC curve and calibrating it with various slope values. When applied to pure phenacetin, a slope of 
$-0.1351\left(\mathrm{~mJ} / \mathrm{s}^{2}\right)$ for indium produces a purity value of $99.89 \mathrm{~mol} \%$. Similarly, a phenacetin slope of -0.08749 $\left(\mathrm{mJ} / \mathrm{s}^{2}\right)$ produces a phenacetin purity value of 99.99 mol\%. The difference between these two purity values is large. Therefore, in the purity determination of a sample, an accurate, precise slope, appropriate to the characteristics of the sample, must be used. Furthermore, if the purity of a substance is to be determined with an error no greater than $0.05 \mathrm{~mol} \%$, the slope of the substance must be determined with an error not exceeding $0.02 \mathrm{~mJ} / \mathrm{s}^{2}$.

\section{Packing a sample}

It was found that crimping indium during the measurement had shown the reproducible value. Many organic compounds sublime during a DSC measurement. For this reason they must be sealed in containers especially designed to hold a liquid. Because organic compounds

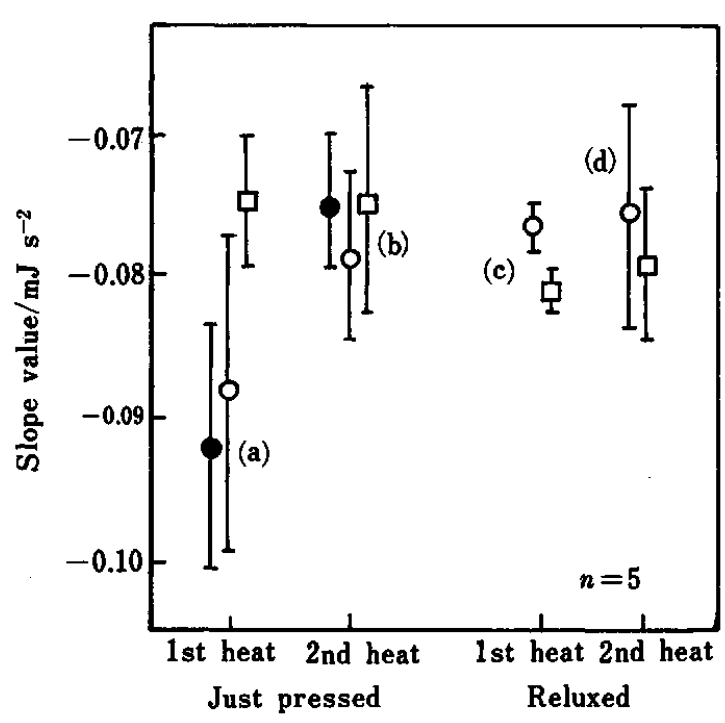

Fig. 2 Comparison regarding the packing of a sample: , no treatment; $O, 100 \mathrm{kgf}$ load; $\square, 200 \mathrm{kgf}$ load. are bulky and have high thermal resistance, samples of such substances are liable to shift in position as they melt, thus producing different DSC curves. ${ }^{5}$ This problem can be solved by pressing the sample onto the bottom of the container. Figure 2 shows the results of experiments conducted using a sealed container.

Using phenacetin as a sample, the effects of the compression pressure on the slope values were investigated. The sample was compressed with a minimum load of $100 \mathrm{kgf}$ in a liquid sample container (Fig. 2 (c)). By contrast, when a sample immediately after compression (Fig. 2 (a)) or being reheated (Fig. 2 (b) and (d)) was used, there was a decline in the reproducibility of the slope of the DSC curve. This was caused by warping in the crystals when the sample was compressed (Fig. 2 (a)). To alleviate this problem, the sample must be left undisturbed for a longer period of time to allow it to loosen. When the sample was reheated, the substance which melted during the first heating process (Fig. 2 (d)) became a small lump, due to its surface tension, and thus changed shape.

When the DSC curve of indium was measured by using the method depicted in Fig. 2 (c), highly reproducible results were obtained, similar to the case in which a crimped container was used. Table 2 shows a comparison between the results obtained using crimped and sealed containers. Whereas almost half of the organic samples underwent changes in quality ${ }^{6}$ during the first heating, the method depicted in Fig. 2 (c) produces favorable results after just one heating, and do not require preheating. This, in the authors' opinion, is valuable information.

\section{Amount of a sample}

Figure 3 shows the influence of the amount of the sample on the slope value of the DSC curve. Experiments were conducted using indium, phenacetin, and sulfonal. Samples weighing less than $1 \mathrm{mg}$ produced gradual, unstable DSC curves. Samples of greater than $2 \mathrm{mg}$ produced highly reproducible results, with stable

Table 1 Change in purity due to the slope value

\begin{tabular}{lccccc}
\hline \multirow{2}{*}{$\begin{array}{c}\text { Reference slope values } \\
\left(\mathrm{mcal} / \mathrm{s}^{2}\right)\end{array}$} & $\left(\mathrm{mJ} / \mathrm{s}^{2}\right)$ & $\begin{array}{c}\text { Pure } \\
(\mathrm{mol} \%)\end{array}$ & $\begin{array}{c}99.3 \\
(\mathrm{~mol} \%)\end{array}$ & $\begin{array}{c}98.0 \\
(\mathrm{~mol} \%)\end{array}$ & $\begin{array}{c}95.0 \\
(\mathrm{~mol} \%)\end{array}$ \\
\hline-0.010 & -0.0418 & 100.04 & 99.38 & 98.18 & 95.56 \\
-0.015 & -0.0628 & 100.01 & 99.40 & 98.18 & 95.53 \\
-0.020 & -0.0837 & 99.99 & 99.33 & 98.30 & 95.57 \\
$-0.0209^{\mathrm{a}}$ & -0.0875 & 99.99 & 99.37 & 98.30 & 98.57 \\
-0.025 & -0.1046 & 99.92 & 99.30 & 98.18 & 95.55 \\
-0.030 & -0.1255 & 99.89 & 99.29 & 98.20 & 95.76 \\
$-0.0323^{b}$ & -0.1351 & 99.91 & 99.30 & 98.20 & 95.53 \\
-0.035 & -0.1464 & 99.92 & 99.27 & 98.14 & 95.46 \\
-0.040 & -0.1674 & 99.94 & 99.26 & 98.17 & 95.48 \\
\hline
\end{tabular}

a. The slope value of phenacetin. Purity value of this line had been calibrated by pure phenacetin.

b. The slope value of indium. Purity value of this line had been calibrated by pure indium. 
DSC curve peaks. Heat absorption by the sample caused the rise in the sample temperature to lag behind the rate increase predicted by the temperature program. The small sample amount ( 2 to $3 \mathrm{mg}$ ) and the slow temperature rise $(1 \mathrm{~K} / \mathrm{min})$ seem to be effective in maximizing the reproducibility of results. This is the method used in most conventional applications. ${ }^{7}$

\section{Thermal conductivity of a sample}

The slope of a DSC curve changes as a function of the thermal resistance of the sample container as well as the interior of the sample, itself. Figure 4 shows the results of an investigation regarding the change in the slope caused by differences in the thermal conductivity of the samples.

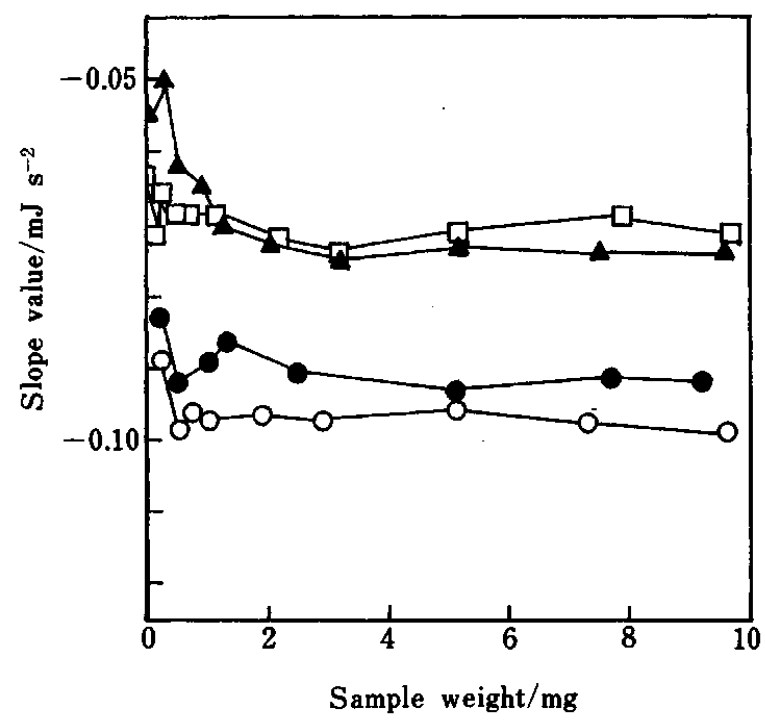

Fig. 3 Effect of the sample weight on the slope of the DSC curve: $\bigcirc$, indium in crimped container; $\boldsymbol{O}$, indium in sealed container; $\boldsymbol{\Delta}$, phenacetin; $\square$, sulfonal.
In this experiment, very pure samples of known thermal conductivity ${ }^{8}$ were used. The logarithm of thermal conductivities was plotted on the $X$-axis, and the slopes of the DSC curves on the $Y$-axis. When plotted on these coordinates, the observed values produced a straight line.

When a liquid container with a substantially bent cover was used to hold liquid samples', DSC curves with high degrees of reproducibility resulted. To obtain the best results it is desirable that the data points for indium and naphthalene (substances that can be obtained in pure form and thermal conductivity) are plotted on semi-log graph paper and then connected by a straight line.

This experiment produced the following straight regressional line:

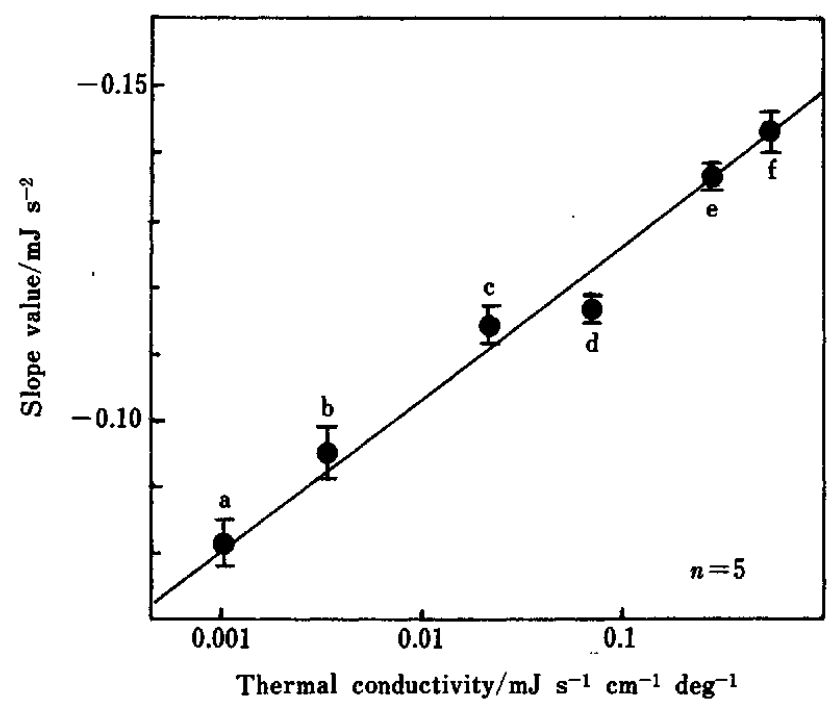

Fig. 4 Slope value of the DSC curves and the thermal conductivity: $a$, benzene; $b$, naphthalene; $c$, ice; $d$, bismuth; $\mathrm{e}$, indium; $\mathrm{f}$, tin.

Table 2 Comparison of the effects of the sample container on DSC curves

\begin{tabular}{|c|c|c|c|c|c|}
\hline & Element & $N$ & Mean & SD & RSD, $\%$ \\
\hline \multirow[t]{4}{*}{ (A) } & Crimped contain & ind & & & \\
\hline & Melt. pt. ${ }^{a}$ & 5 & 155.18 & 0.05 & \\
\hline & Heat of fusion ${ }^{b}$ & 5 & 29.288 & 0.2677 & 0.91 \\
\hline & Slope ${ }^{c}$ & 5 & -0.13196 & 0.00163 & 1.23 \\
\hline \multirow[t]{4}{*}{ (B) } & Sealed container & diu & & & \\
\hline & Melt. pt. ${ }^{\mathrm{a}}$ & 5 & 155.18 & 0.08 & \\
\hline & Heat of fusion ${ }^{b}$ & 5 & 29.405 & 0.1799 & 0.61 \\
\hline & Slope $^{c}$ & 5 & -0.12577 & 0.00552 & 4.38 \\
\hline \multirow[t]{4}{*}{ (C) } & Sealed container & ted & mple: indiur & & \\
\hline & Melt. pt. ${ }^{\mathrm{a}}$ & 5 & 154.90 & 0.00 & \\
\hline & Heat of fusion ${ }^{b}$ & 5 & 27.438 & 0.2803 & 1.02 \\
\hline & Slope $^{c}$ & 5 & -0.12100 & 0.00192 & 1.59 \\
\hline \multirow[t]{4}{*}{ (D) } & Sealed container & tec & mple: phena & & \\
\hline & Melt. pt. ${ }^{\mathrm{a}}$ & 5 & 132.68 & 0.11 & \\
\hline & Heat of fusion ${ }^{b}$ & 5 & 161.703 & 1.3012 & 0.80 \\
\hline & Slope $^{c}$ & 5 & -0.07736 & 0.00163 & 2.10 \\
\hline
\end{tabular}

a. ${ }^{\circ}$ C. b. $\mathrm{mJ} / \mathrm{mg}$. c. $\mathrm{mJ} / \mathrm{s}^{2}$. 


$$
Y=-0.02237 \log X-0.1476 \text {, }
$$

where $X$ is the thermal conductivity $(\mathrm{mJ} / \mathrm{s} \cdot \mathrm{cm} \cdot \mathrm{K})$ and $Y$ is the slope of the curve $\left(\mathrm{mJ} / \mathrm{s}^{2}\right)$.

As the above equation indicates, a tolerance limit of $0.02 \mathrm{~mJ} / \mathrm{s}^{2}$ for the slope of a DSC curve is equivalent to an order-of-magnitude difference in the thermal conductivities.

Thus, using this straight line, the slope of a DSC calibration curve can be obtained from the thermal conductivity of a given sample.

\section{Determination of purity}

Table 3 shows the results of the measurement of an

Table 3 Analytical results of phenacetin

\begin{tabular}{lcccc}
\hline \multicolumn{5}{c}{ Purity of sample (mol\%) } \\
& $99.92 \pm 0.2$ & $99.2 \pm 0.2$ & $98.0 \pm 0.2$ & $94.9 \pm 0.5$ \\
\hline & 99.94 & 99.34 & 98.31 & 95.60 \\
& 99.91 & 99.35 & 98.33 & 95.79 \\
& 99.93 & 99.36 & 98.27 & 95.58 \\
& 99.91 & 99.32 & 98.26 & 95.57 \\
$N$ & 99.94 & 99.35 & 98.26 & 95.80 \\
Mean & 99.93 & 99.34 & 98.29 & 95.67 \\
Bias & +0.0 & +0.1 & +0.3 & +0.8 \\
SD & 0.02 & 0.02 & 0.03 & 0.12 \\
\hline
\end{tabular}

NBS purity set. Phenacetin with a purity of $99.2 \pm$ $0.2 \mathrm{~mol} \%$ was determined with an error no greater than $0.1 \mathrm{~mol} \%$; similarly, phenacetin with a purity of $98.0 \pm$ $0.2 \mathrm{~mol} \%$ was determined with an error no greater than $0.3 \mathrm{~mol} \%$. However, a phenacetin sample with a $94.9 \pm 0.5 \mathrm{~mol} \%$ purity did not yield an acceptable value, because it contained impurities beyond the limits imposed by the van't Hoff's equation. Table 4 gives the results of tests conducted on mixed samples designed to contain 1 to $2 \mathrm{~mol} \%$ of impurities. Table 4 also shows the results of measurements by microanalysis and HPLC. These values agree markedly with the results of the DSC method. Other analytical methods also agreed noticeably with the computed values.

The authors investigated the conditions to be employed for the determination of purity.

The sample is pressed onto the bottom of a sample container by a mold. Highly reproducible DSC curves were obtained after calibrating for the lag in the temperature rise due to the thermal resistance of the sample. When used for the purity determination of highly pure samples, this method produces results having great precision, accuracy, reliability, and minimal error.

\section{References}

1. Sub-committee for micro analytical standards of the microchemistry group of the society for analytical

Table 4 Analytical results

\begin{tabular}{|c|c|c|c|c|c|c|}
\hline \multirow{2}{*}{ Material $^{\mathrm{a}}$} & \multicolumn{4}{|c|}{ Purity $(\mathrm{mol} \%)^{b}$} & \multicolumn{2}{|c|}{ Microanalysis $(\%)^{d}$} \\
\hline & Calcd. & DSC & Error & HPLC $^{c}$ & Calcd. & Found \\
\hline \multirow{4}{*}{$\begin{array}{l}m \text {-Dinitrobenzene } \\
\text { (1-Chloro-2,4- } \\
\text { dinitrobenzene) }\end{array}$} & 98.13 & 98.12 & -0.01 & 98.42 & $\mathrm{Cl}=0.39$ & 0.39 \\
\hline & 98.48 & 98.47 & -0.01 & 98.40 & 0.31 & 0.32 \\
\hline & 99.04 & 99.04 & 0.00 & 98.70 & 0.20 & 0.20 \\
\hline & 99.50 & 99.50 & 0.00 & 99.45 & 0.10 & 0.10 \\
\hline \multirow{4}{*}{$\begin{array}{l}\text { Acetanilide } \\
\text { ( } p \text {-Bromo - } \\
\text { acetanilide) }\end{array}$} & 97.98 & 97.87 & -0.11 & 97.91 & $\mathrm{Br}=1.17$ & 0.19 \\
\hline & 98.49 & 98.50 & +0.01 & 98.47 & 0.88 & 0.90 \\
\hline & 99.07 & 99.07 & 0.00 & 99.11 & 0.54 & 0.56 \\
\hline & 99.52 & 99.52 & 0.00 & 99.46 & 0.28 & 0.29 \\
\hline \multirow{4}{*}{$\begin{array}{l}\text { Benzoic acid } \\
\text { ( } m \text {-Dinitro- } \\
\text { benzene) }\end{array}$} & 98.02 & 98.02 & 0.00 & 98.05 & $\mathrm{~N}=0.45$ & 0.45 \\
\hline & 98.50 & 98.49 & -0.01 & 98.50 & 0.34 & 0.34 \\
\hline & 99.00 & 98.99 & -0.01 & 98.75 & 0.21 & 0.22 \\
\hline & 99.52 & 99.50 & -0.02 & 99.61 & 0.10 & 0.11 \\
\hline \multirow{4}{*}{$\begin{array}{l}p \text {-Nitroaniline } \\
\text { (o-Nitroaniline) }\end{array}$} & 98.12 & 98.12 & 0.00 & 98.39 & & \\
\hline & 98.51 & 98.49 & -0.02 & 98.86 & & \\
\hline & 99.00 & 98.98 & -0.02 & 99.07 & & \\
\hline & 99.50 & 99.51 & +0.01 & 99.25 & & \\
\hline \multirow{4}{*}{$\begin{array}{l}\text { Anthracene } \\
\text { (Pyrene) }\end{array}$} & 98.09 & 97.97 & -0.12 & 98.06 & & \\
\hline & 98.53 & 98.50 & -0.03 & 98.66 & & \\
\hline & 99.00 & 98.99 & -0.01 & 99.03 & & \\
\hline & 99.56 & 99.55 & -0.01 & 99.39 & & \\
\hline
\end{tabular}

a. Chemicals in parenthesis are minor component. b. Mol\% of major component on mixed. Mean value of 2 runs. c. Ratio of peak area. Mean value of 2 runs. d. Weight $\%$ of element arised from minor component. Mean value of 2 runs. 
chemistry, Analyst [London], 87, 304 (1962).

2. M. E. Brown, "Introduction to Thermal Analysis", p.152, Chapman \& Hall, New York, 1988.

3. S. Takagi: Netsu Sokutei, 9, 124 (1982).

4. K. Narita and H. Kitamura, Bunseki Kagaku, 35, 303 (1986).

5. K. Yamamoto and K. Narita: The Rigaku-Denki J., 18, 15 (1987).

6. K. Yamamoto, H. Kitamura, K. Narita, T. Itakura and M. Momota, Abstracts Proceeding of 24th Japanese Conference on Calorimetry and Analysis (1988), p.216.
7. R. L. Blaine, C. K. Schoff (ed.), "Purity Determinations by Thermal Methods", ASTM Special Technical Publication, p.31 (1983).

8. J. A. Dean: "Lange's Handbook of Chemistry", ed.11, 3-2, 10-274, McGraw-Hill Book Co., New York, 1973.

9. Mettler Instrumente A.G: “Mettler TA4000 System Operating Instruction", p. 27 (1988).

(Received February 3, 1992)

(Accepted April 27, 1992) 